\title{
MtENOD20, a Nod Factor-Inducible Molecular Marker for Root Cortical Cell Activation
}

\author{
Vanessa Vernoud, Etienne-Pascal Journet, and David G. Barker \\ Laboratoire de Biologie Moléculaire des Relations Plantes-Microorganismes, INRA-CNRS UMR215, BP 27, \\ 31326 Castanet-Tolosan Cedex, France \\ Accepted 27 February 1999.
}

\begin{abstract}
The spatio-temporal expression pattern of the Medicago truncatula ENOD20 gene during early stages of nodulation has been analyzed with transgenic alfalfa (M. varia) expressing a pMtENOD20-GUS chimeric fusion. Our results show that transcriptional activation of this gene occurs initially in dividing inner cortical cells corresponding to sites of nodule primordium formation and subsequently in root hairs containing infection threads. Use of Sinorhizobium meliloti nod gene mutants that uncouple nodule organogenesis from infection has confirmed that early MtENOD20 transcription is tightly linked to cortical cell activation (CCA). Furthermore, these experiments have revealed that an $S$. meliloti nodH mutant, defective in Nod factor sulfation and epidermal cell activation, is nevertheless able to elicit low-level CCA. Purified $S$. meliloti Nod factors trigger MtENOD20 transcription very rapidly (within 12 to $24 \mathrm{~h}$ ) in the root cortex, and studies with transgenic alfalfa show that Nod factors are able to elicit gene expression coupled to $\mathrm{CCA}$ at concentrations as low as $10^{-11} \mathrm{M}$. Finally, we have made use of a range of synthetic lipo-chitooligosaccharides to show that fatty acid chain length is an important structural parameter in the capacity of Nod factors to elicit CCA. Taken together, our results suggest that pMtENOD20-GUS transgenic lines should prove valuable tools in future studies of Rhizobium and Nod factor-elicited CCA.
\end{abstract}

Additional keywords: early nodulin, GUS reporter gene.

The symbiotic association between leguminous plants and soil bacteria known as rhizobia leads to the formation of complex root structures (nodules) in which the bacteria reduce atmospheric nitrogen to ammonia, thus providing the host plant with a privileged source of combined nitrogen. Early stages of root nodulation involve developmental host responses that are related either to root penetration by the bacterium (bacterial infection) or to the initiation of nodule organogenesis within the cortex. These responses result from a molecular dialogue between the two organisms, and in particular the synthesis of specific lipo-chitooligosaccharide (LCO) signaling molecules (known as Nod factors) by the microsymbiont (for recent reviews see Dénarié et al. 1996; Heidstra and Bisseling 1996; Long 1996). Nod factors possess multiple biological activities

Corresponding author: David G. Barker

E-mail: barker@toulouse.inra.fr that are related either to rhizobial infection (e.g., hair deformation) or nodule organogenesis (e.g., cortical cell activation [CCA]), and these molecules are of fundamental importance to successful host/bacterial recognition and root nodulation.

One of the best-studied $\mathrm{N}_{2}$-fixing symbiotic associations is that formed between Sinorhizobium meliloti and Medicago species such as alfalfa or the model legume M. truncatula. S. meliloti Nod factors (NodRm) consist of a chito-tetraose backbone, C6-sulfated at the reducing end and both $\mathrm{O}$ acetylated and $\mathrm{N}$-acylated (majority $\mathrm{C} 16: 2$ fatty acid) at the non-reducing end (Lerouge et al. 1990). It has been shown that purified NodRm elicits both root hair deformation in alfalfa at concentrations as low as $10^{-12} \mathrm{M}$ (Roche et al. 1991) and CCA leading to visible root outgrowths down to $10^{-9} \mathrm{M}$ (Truchet et al. 1991). Use of both $S$. meliloti nod gene mutants (nod genes encode the enzymes responsible for Nod factor synthesis) and purified, structurally altered Nod factors has revealed that substitutions at both ends of the NodRm sugar backbone are important for biological activity (reviewed in Dénarié et al. 1996).

The identification of plant genes (encoding so-called early nodulins) that are activated during the early stages of root infection/nodulation can facilitate the study of the molecular and cellular responses of the plant to rhizobial Nod factors (see review in Heidstra and Bisseling 1996). With this goal in mind we recently described the initial characterization of two new early nodulin genes (MtENOD16 and MtENOD20), isolated by low-stringency screening of an M. truncatula genomic library with a pea ENOD5 cDNA probe (Greene et al. 1998). Sequence analysis showed that both of these genes encode extracellular proteins belonging to a small family of early nodulins (including Ps/VsENOD5 and GmN315/ ENOD55) with structural homology to copperbinding phytocyanins.

In this article, we present a detailed study of the nodulationrelated expression pattern of the MtENOD2O gene, making use of transgenic alfalfa lines into which we have introduced a chimeric gene consisting of a $2.1-\mathrm{kb}-$ long MtENOD20 promoter fragment fused to the $\beta$-glucuronidase $(G U S)$ reporter gene. We show that, following $S$. meliloti inoculation, transcription of the MtENOD2O gene is initially induced in the root inner cortex at sites of nodule primordium formation, and subsequently during host cell infection. We have exploited these transgenic lines, in combination with S. meliloti nod gene mutants and purified Nod factors (both biological and synthetic), to establish the relationship between MtENOD20 
gene expression and CCA and to demonstrate the utility of this new molecular marker in Nod factor structure/function studies.

\section{RESULTS}

\section{Construction of the pMtENOD20-GUS fusion and introduction into Medicago varia $\mathrm{A} 2$.}

In order to study the spatio-temporal expression pattern of the MtENOD20 gene during both early and late stages of nodulation, we constructed a transcriptional fusion between the 2.1-kb DNA fragment lying immediately upstream of the MtENOD20 ATG initiation codon and the coding region of the GUS reporter gene (see Materials and Methods). This chimeric gene was then cloned into the binary vector pLP100 (Szabados et al. 1995) and introduced via Agrobacterium tumefaciens-mediated leaf-disk transformation into the highly embryogenic alfalfa Medicago varia A2 (Deak et al. 1986). A total of 22 independent kanamycin-resistant transgenic lines were regenerated following somatic embryogenesis of transformed callus tissue. Primary transformants were then grown in aeroponic chambers and inoculated with Sinorhizobium meliloti RCR2011 (Rosenberg et al. 1981). Preliminary analyses of root and nodule tissues at various times after inoculation revealed qualitatively identical patterns of GUS activity for 17 out of the 22 lines. Tissues from the remaining 5 lines were negative for GUS staining. The detailed studies described below were performed with representative lines that expressed high levels of the pMtENOD20-GUS fusion. $\mathrm{S}_{1}$ seed obtained by self-fertilization was used for experiments in growth pouches.

\section{Expression of MtENOD20 during $S$. meliloti root infection and nodulation.}

Prior to inoculation, GUS activity was undetectable in cortical, epidermal, and meristematic root tissues of transgenic pMtENOD20-GUS lines. On the other hand, weak staining was occasionally observed in root vascular tissues of 10-dayold, pouch-grown plants. Apart from the root, the only transgenic plant tissue in which we have been able to detect GUS activity is the seed endosperm (data not shown).

Following inoculation with $S$. meliloti RCR2011, expression of the pMtENOD20-GUS fusion could first be detected in the root cortex after approximately 24 to $36 \mathrm{~h}$ (Fig. 1A). At this stage, root hair infection was not yet detectable and GUS staining was strictly limited to discrete cortical loci within the nodulation-susceptible region of the root (see Materials and Methods). Thin sectioning revealed that reporter gene expression was associated with localized regions of cell division within the root inner cortex (Fig. 1B), corresponding to nodule primordium development. The size and intensity of these GUS staining regions increased significantly by 48 to $72 \mathrm{~h}$ post inoculation (hpi), with the additional appearance of reporter gene activity in associated root hairs displaying shepherd's crook curling, characteristic of root hair infection (Fig. 1C and D). Sections through stained cortical regions $72 \mathrm{hpi}$ again showed that GUS activity was present in regions of nodule development (Fig. 1E). Note that the MtENOD20 gene is not expressed in tissues of emerging lateral root primordia (Fig. 1E). Finally, observation of many root systems harvested and stained for GUS activity between 24 and 48 hpi have confirmed that MtENOD20 expression in the cortex always precedes infection-related expression in root hairs.

At the stage when nodules became clearly visible as root outgrowths (approximately 4 days post inoculation) GUS staining was very intense in the central tissues of these young developing nodules, which mainly consist of cells undergoing infection by the highly ramified infection thread network (Fig. $1 F)$. Adjacent to these emerging nodules, GUS staining was also clearly visible at localized sites in peripheral root tissues (Fig. 1F and G). Double-staining for $\beta$-galactosidase activity to detect the presence of the bacterium (see Materials and Methods) showed that infection threads were present in the epidermal GUS-staining cells (Fig. 1H). Note that the red coloring of the infection thread is masked by the overlaying blue staining of the GUS activity in the cytoplasm. We presume that these events correspond to unsuccessful infections in which progression of the infection thread has been arrested either in epidermal or outer cortical cell layers, as previously reported by Penmetsa and Cook (1997) and Vasse et al. (1993), respectively.

Sectioning and subsequent staining of mature nitrogenfixing nodules (Fig. 1I) revealed that reporter gene activity is present exclusively in central tissues. GUS staining is totally absent in nodule meristematic tissue, and can first be detected in distal zone II where infection initiates, increasing in intensity as infected cells differentiate and enlarge toward the proximal end of this zone (for the definition of nodule zones see Vasse et al. 1990). Following the amyloplast-rich interzone II-III, staining becomes generally weak and patchy throughout the nitrogen-fixing zone III, and at present we do not know the reason for this irregular staining pattern.

Various attempts were made to confirm that the endogenous MtENOD20 gene is activated rapidly in the M. truncatula root following $S$. meliloti inoculation. Unfortunately, both Northern (RNA) analysis (with total RNA extracts) and in situ hybridization proved insufficiently sensitive to detect MtENOD2O transcript induction during the first 24 to $48 \mathrm{hpi}$. We therefore made use of the reverse transcriptase-polymerase chain reaction (RT-PCR) approach, and to maximize sensitivity PCR was performed with specific primers located on either side of the single MtENOD2O intron, thus allowing us to distinguish between amplified fragments of genomic or cDNA origin (see Materials and Methods). The RT-PCR experiment illustrated in Figure 2A shows that MtENOD20 mRNA is absent in noninoculated $M$. truncatula roots, and can first be detected (albeit at a low level) as early as 24 hpi with $S$. meliloti RCR2011. This and the subsequent increases in transcript levels observed 48 to 72 hpi correlate well with the timing and extent of reporter gene activity observed in the transgenic $M$. varia expressing $\mathrm{p} M t E N O D 20-G U S$. The quality of the cDNAs used in the various PCRs was controlled by performing parallel amplifications with primers specific for the constitutively expressed MtPR10-1 gene (Gamas et al. 1998; and see Materials and Methods).

In conclusion, our results argue that expression of the MtENOD20 gene can be linked to at least two distinct early stages of nodulation. Expression is first detected during nodule primordium formation in the root inner cortex, and then subsequently in both root hairs and nodule tissues that contain infection threads. 

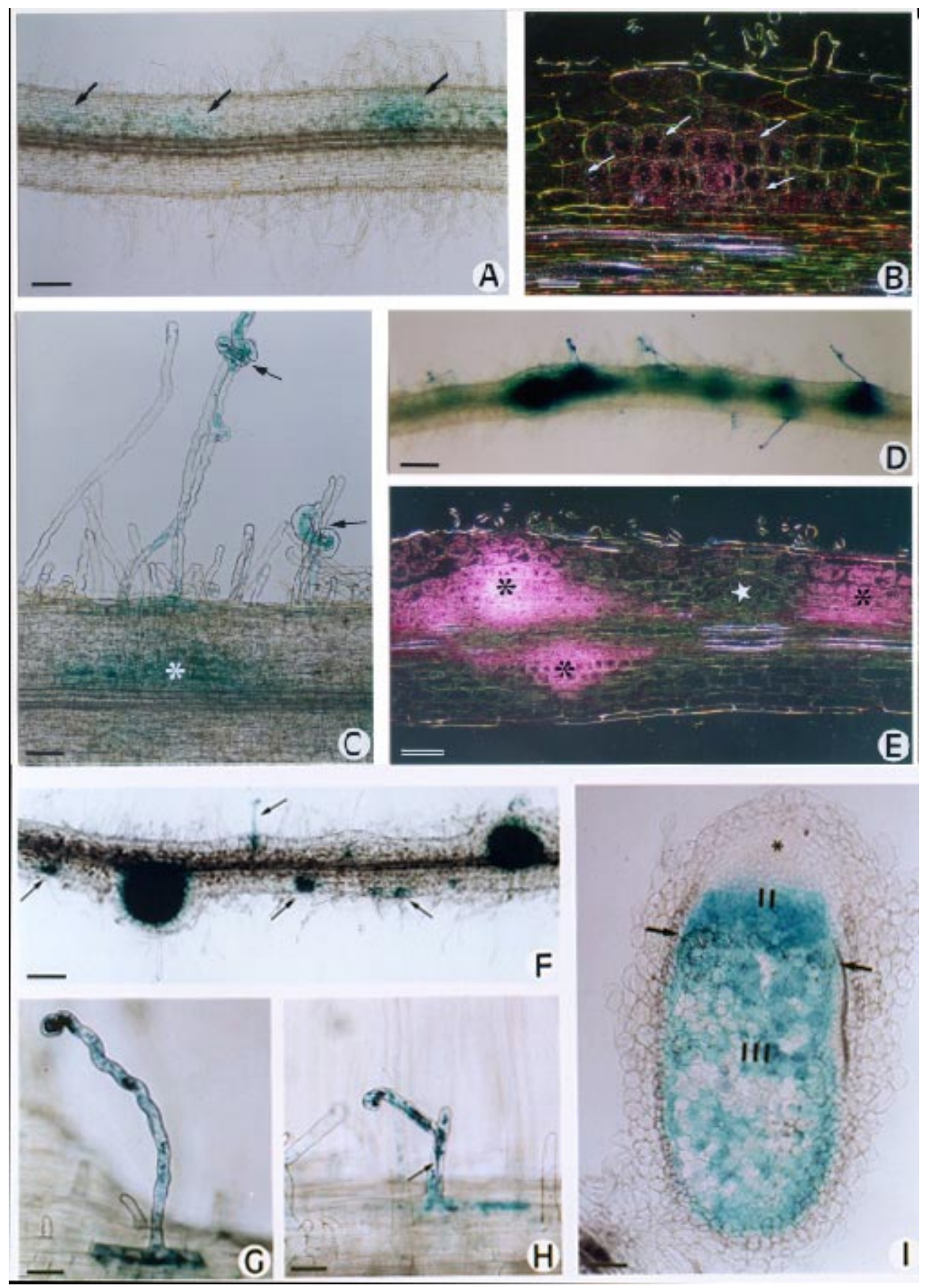

Fig. 1. Histochemical localization of $\beta$-glururonidase (GUS) activity throughout various stages of root nodulation in transgenic Medicago varia expressing the pMtENOD20-GUS fusion. A, Whole root segment close to the root tip $36 \mathrm{~h}$ post inoculation (hpi) with Sinorhizobium meliloti RCR2011. Arrows indicate cortical sites staining positively for GUS activity. There is an increase in staining intensity as the root develops (zone of root hair emergence is at left-hand end of the segment). Bar $=100 \mu \mathrm{m}$. B, Section ( $4 \mu \mathrm{m}$ thick) of a Technovit-embedded root 36 hpi. GUS staining (colored red under dark-field observation) is localized mainly to inner cortical cell layers. Arrows indicate anticlinal divisions. Bar $=25 \mu \mathrm{m}$. C, Whole root segment $48 \mathrm{hpi}$. GUS activity is visible in the cortex (asterisk) and in associated root hairs with characteristic shepherd's crook curling (arrows). Bar $=50 \mu \mathrm{m}$. D, Stereomicroscopic image of whole root segment $72 \mathrm{hpi}$ illustrating relationship between GUS staining in the cortex and in associated infected root hairs. Bar $=200 \mu \mathrm{m}$. E, Longitudinal root section (4 $\mu \mathrm{m}$ thick) $72 \mathrm{hpi}$ viewed under dark-field optics. GUS activity (red coloration) localizes to developing nodule primordia (asterisks), but is totally absent in a lateral root primordium (star). Bar $=50 \mu \mathrm{m}$. F, Whole root segment 4 days post inoculation showing intense GUS staining in inner tissues of emerging nodules. Arrows indicate staining in peripheral tissues corresponding to arrested infection events. Bar $=200 \mu \mathrm{m}$. G, GUS staining in infected root hair where infection thread progression has arrested within the epidermal cell. Bar $=50 \mu \mathrm{m} . \mathbf{H}, \mathrm{As}$ for $\mathrm{G}$, except that tissue has been double-stained for GUS (blue) and $\beta$-galactosidase (red) to localize the bacteria (expressing the constitutive hemA-lacZ fusion) in the infection thread (arrow). The red coloring is masked by the overlying blue GUS staining. Bar $=50 \mu \mathrm{m}$. I, Longitudinal section $(80 \mu \mathrm{m}$ thick) through a mature 10-day-old nitrogen-fixing nodule. GUS staining is confined to central tissues of the nodule and extends from infection zone II down through nitrogen-fixing zone III. The nodule meristem is marked by an asterisk; arrows indicate amyloplast-rich interzone II-III layer. Bar $=100 \mu \mathrm{m}$. 
CCA and associated MtENOD20 gene expression elicited by $S$. meliloti nodH and nodF/nodL mutants.

Mutations that inactivate key genes within the S. meliloti nodulation (nod) locus result in modifications to the structure of the symbiotic Nod factor molecule and thereby generate mutants with a variety of infection/nodulation phenotypes. Certain of these mutants are reported to entirely lack the capacity to induce symbiotic responses in the host plant, whereas others elicit modified epidermal/cortical responses. In order to further evaluate the relationship between MtENOD20 expression and early stages of nodulation we inoculated transgenic alfalfa plants expressing the pMtENOD20-GUS fusion with various $S$. meliloti nod mutants.

As a control we initially tested a strain with a polar mutation in the $\operatorname{nod} A$ gene. This mutation abolishes synthesis of all three enzymes encoded by the nodABC operon, together responsible for elaborating the Nod factor LCO core structure (reviewed in Dénarié et al. 1996). As expected, inoculation with the nodA mutant failed to elicit expression of the MtENOD20 gene in either epidermal or cortical tissues (Table 1). The second type of $S$. meliloti strain tested was mutated in the host-specificity gene nodH. Nod factors synthesized by a nodH mutant lack a critical sulfate substitution on the C-6 position at the reducing end of the sugar backbone (Roche et al. 1991). A variety of bioassays have previously shown that nodH mutants are unable to elicit ei-

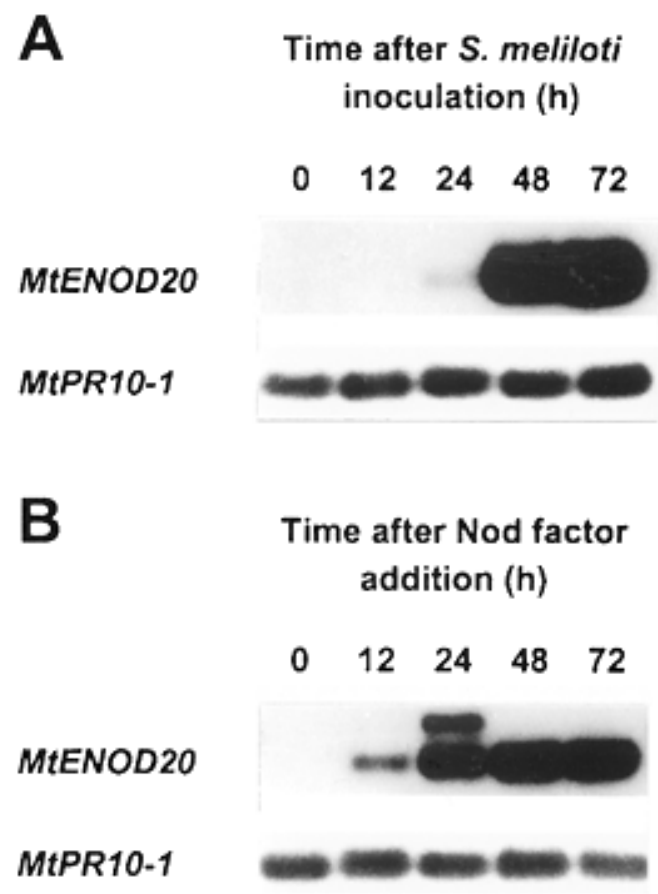

Fig. 2. Transcriptional activation of MtENOD20 in Medicago truncatula roots following Sinorhizobium meliloti inoculation and Nod factor addition. M. truncatula plants in growth pouches were (A) inoculated with $S$. meliloti RCR2011 or (B) treated with $5 \times 10^{-8}$ M NodRm. Secondary roots were harvested at the indicated time points, total RNA extracted, and MtENOD2O transcript levels evaluated by reverse transcriptasepolymerase chain reaction analysis (see Materials and Methods). The additional higher molecular weight band in the 24-h Nod factor-treated sample corresponds to amplification of contaminating genomic DNA (see Materials and Methods). Parallel amplification of MtPR10-1 mRNA was used as a control for quality and quantity of RNA samples. ther symbiotic epidermal responses (root hair curling/MtENOD12 expression) or root nodulation on the normal host plant alfalfa (Debellé et al. 1986; Journet et al. 1994). Nevertheless, reporter gene activity was detected in roots of transgenic alfalfa as early as 48 hpi with a strain carrying a Tn5 insertion in the nodH gene. At 72 hpi, GUS staining was clearly observed as discrete patches in regions of the root normally susceptible to nodulation at the moment of inoculation (see Materials and Methods) and was localized essentially to inner cortical tissues (Fig. 3A). Approximately one third of inoculated plants stained positively (albeit weakly) for inner cortical GUS expression $48 \mathrm{hpi}$, compared with a $100 \%$ response for the wild-type strain (Table 1). From 72 hpi onward, the percentage of plants with detectable GUS activity increased to $80 \%$ with an accompanying increase in the intensity of staining. Detailed observation of the responsive zones at 72 hpi revealed a tight correlation between GUS staining and the presence of both cortical cell divisions and features such as centrally positioned nuclei and amyloplast accumulation (Fig. 3A and data not shown). This type of symbiotic response has been termed cortical cell activation (CCA) and is typically observed under conditions of defective infection, such as that found following inoculation with the nodF/nodL double mutant (Ardourel et al. 1994; see below). Identical results were obtained for a second nodH mutant, this time carrying a deletion within the coding region of the gene (Table 1), confirming that Nod factor sulfation-defective $S$. meliloti mutants are indeed capable of eliciting CCA. Significantly, these regions of CCA do not develop further, and in no case did we observe external swellings of the root. Furthermore, morphological responses such as root hair deformations were not observed in epidermal tissues located above regions of CCA, and reporter gene activity was never detected in root hairs of plants inoculated with nodH mutants. Finally, we have recently observed similar patterns of reporter gene expression in cortical cell layers following $\mathrm{nodH}$ mutant inoculation of $M$. truncatula plants transformed with the identical pMtENOD20-GUS construct (data not shown).

The third $S$. meliloti mutant examined, defective in both the $n o d F$ and nodL genes, produces Nod factors that are doubly modified at the nonreducing end of the sugar backbone. These LCOs lack the $O$-acetyl group and are N-acylated with cisvaccenic acid ( $\Delta 11-\mathrm{C} 18: 1)$ instead of the normal $\Delta 2,9-\mathrm{C} 16: 2$ fatty acid chain (Ardourel et al. 1994). nodF/nodL mutants are defective in infection and nodulation, but nevertheless are able to elicit striking multiple root hair deformations and an extensive CCA response (Ardourel et al. 1994). Figure 3B shows a GUS-stained segment of a transgenic $M$. varia root 4 days after inoculation with a nodF/nodL mutant. Consistent with our

Table 1. Percentage of $\beta$-glucuronidase (GUS)-positive transgenic plants following inoculation with Sinorhizobium meliloti nod mutants

\begin{tabular}{lcccc}
\hline & \multicolumn{4}{c}{ Time following inoculation (days) } \\
\cline { 2 - 5 } S. meliloti strain & $\mathbf{2}$ & $\mathbf{3}$ & $\mathbf{5}$ & $\mathbf{1 0}$ \\
\hline RCR2011 (parent) & 100 & 100 & 100 & 100 \\
RCR2011 nodA::Tn5 & 0 & 0 & 0 & 0 \\
RCR2011 nodH::Tn5 & 30 & 80 & 80 & 80 \\
RCR2011 $\Delta$ nodH & 20 & 80 & 80 & 80 \\
RCR2011 $\Delta$ nodF/nodL::Tn5 & 40 & 70 & 90 & 100 \\
\hline
\end{tabular}


findings for the nodH mutants, regions showing clear CCA responses also stained intensely for GUS activity. At this stage, no staining was observed in the highly deformed root hairs adjacent to CCA-responsive regions of the root (Fig. 3B), in line with the infection-defective phenotype of this mutant (Ardourel et al. 1994). Despite similar kinetics for the percentage of plants responding to nodH and nodF/nodL mutants following inoculation (Table 1), it should be emphasized that the cortical response to the nodF/nodL mutant was generally more extensive and more intense with regard to GUS staining, and that regions of CCA elicited by this mutant often led to visible root swellings 7 to 10 days after inoculation (data not shown).

\section{MtENOD20 expression in root tissues in response to $S$. meliloti Nod factors.}

Our results with wild-type and mutant strains suggested that MtENOD20 expression could serve as a useful marker for early symbiotic responses, and in particular those involving CCA. Since purified Nod factors are capable of eliciting root nodule organogenesis in alfalfa (Truchet et al. 1991), we decided to examine Nod factor responses in roots of transgenic plants expressing $\mathrm{p} M t E N O D 20-G U S$. Initial experiments were carried out with $10^{-8} \mathrm{M}$ NodRm, since relatively high concentrations of the $S$. meliloti Nod factor $\left(10^{-9}\right.$ to $\left.10^{-7} \mathrm{M}\right)$ are required to observe nodulation-related root deformations (Truchet et al. 1991). We found that reporter gene activity could be readily detected in inner cortical root tissues 24 to 48 $\mathrm{h}$ following addition of $10^{-8} \mathrm{M}$ NodRm to transgenic plants grown in pouches (Fig. 3C). GUS staining localized to zones within the root corresponding to the nodulation-susceptible region of the root at the moment of Nod factor addition. Figure $3 \mathrm{C}$ also clearly illustrates the typical Nod-factor-induced root hair deformations (most frequently hair branchings), which occur in regions of the epidermis located above the zones of cortical staining. Note that GUS activity is not associated with these root hair deformations, again consistent with our conclusion that MtENOD20 expression in root hairs is dependent upon infection thread formation. Detailed observations of the GUS-staining cortex revealed the presence of both cortical cell divisions and starch-containing cells (data not shown), underlining once more the relationship between symbiotic CCA and MtENOD20 expression. Approximately two thirds of pouch-grown plants scored positive for cortical GUS staining as early as $24 \mathrm{~h}$ following addition of $10^{-8} \mathrm{M}$ NodRm, with the percentage of positively staining plants increasing to $100 \% 48$ h later (Table 2).

The kinetics of Nod-factor-dependent MtENOD20 mRNA accumulation were then examined with the sensitive RT-PCR approach described above. Figure 2B shows that MtENOD2O transcripts can be detected within $12 \mathrm{~h}$ following addition of 5 $\times 10^{-8} \mathrm{M}$ NodRm to $M$. truncatula roots. Differences in the timing of mRNA accumulation elicited by $S$. meliloti and purified Nod factors (compare Figure 2A and B) are probably due to the relatively high concentration of NodRm used in this experiment and to the fact that the Nod factors must first be synthesized in the bacteria.

The rapidity of $\mathrm{p} M t E N O D 20-G U S$ gene activation in the cortex in response to $10^{-8} \mathrm{M}$ NodRm encouraged us to investigate the reactivity of the root cortex to much lower concentrations of Nod factor. Dose-response experiments with
MtENOD20 expression as a qualitative measure of CCA showed that Nod factors are in fact active down to $10^{-11} \mathrm{M}$, although the percentage of responding plants is significantly lower at subnanomolar concentrations of the LCO elicitor (Table 2). Note that only differences of greater than $10 \%$ in the percentage of GUS-staining plants can be considered statistically significant (see Materials and Methods). We also observed that the extent and intensity of individual responding regions were generally reduced at these low Nod factor concentrations.

\section{CCA elicited by modified Nod factors.}

It has previously been shown that modifications to the structure of the $S$. meliloti Nod factor can result in significant alterations in the capacity of this signaling molecule to elicit visible, nodulation-related root deformations in the alfalfa root (Truchet et al. 1991). We therefore attempted to use transgenic pMtENOD20-GUS alfalfa plants as a sensitive semiquantitative assay system for evaluating the effects of such structural modifications on early cortical cell responses. For comparative purposes all Nod factor variants were tested at a concentration of $10^{-8} \mathrm{M}$ and reporter gene expression was monitored during a period of 2 to 5 days after addition of the elicitor. Following the earlier experiments with nodF/nodL and nodH mutants, we initially tested the corresponding modified factors purified from these two strains. As expected, and in line with results previously presented by Ardourel et al. (1994) the non-O-acetylated cisvaccenic acid factor produced by the nodF/nodL mutant was indeed able to elicit cortical MtENOD20 gene expression (Table 3). On the other hand, and rather to our surprise, we were unable to detect reporter gene activity in roots of transgenic plants treated with the purified nonsulfated factor synthesized by the nodH mutant. This negative result was confirmed by testing a total of more than 30 plants in several independent experiments and with a higher (up to $10^{-7}$ M) nonsulfated factor concentration (data not shown). Different hypotheses explaining this discrepancy between the CCA-inducing activities of the nodH mutant and the purified nonsulfated factor will be discussed later.

Since removing the $O$-acetyl group and replacing the fatty acid C16:2 by C18:1 did not appear to be critical for Nodfactor-dependent CCA we decided to vary the length of the fatty acid chain. In these experiments we made use of a range of chemically synthesized, O-sulfated LCOs lacking the $O$ acetyl group and with linear mono-unsaturated fatty acid chains ranging from 0 to 16 carbon atoms in length (DemontCaulet et al., in press). The results presented in Table 3 show that a major reduction in the percentage of reactive plants was observed when the fatty acid chain was shortened to half the normal length. As with very low concentrations of NodRm (see above), the extent and intensity of the responding regions were also reduced in response to $\mathrm{C} 8: 1 \mathrm{~N}$-acylated molecules. When the fatty acid moiety was totally removed, leaving a sulfated chitin tetramer [CO-IV $\left(\mathrm{S}, \mathrm{NH}_{2}\right)$ ], we observed a total loss of CCA activity (Table 3 ), and it was subsequently confirmed that this is also the case at the higher concentration of $10^{-7} \mathrm{M}$ (data not shown). These results suggest that the presence and length of the acyl chain play a key role in the capacity of the $S$. meliloti Nod factor to elicit the CCA response in alfalfa. 


\section{DISCUSSION}

\section{The MtENOD20 gene is transcribed during both nodule} organogenesis and host cell infection.

The expression pattern of the M. truncatula ENOD20 early nodulin gene during root nodulation has been studied at the cellular level with $M$. varia plants transformed with a promoter-GUS gene fusion. The validity of such a transgenic plant approach has already been demonstrated for several other early nodulin genes including ENOD12 (Pichon et al. 1992; Bauer et al. 1996), ENOD40 (Fang and Hirsch 1998), and Mtlecl\&3 (Bauchrowitz et al. 1996), and is particularly appropriate when gene expression is limited to discrete subpopulations of cells within a given organ. Our results show that the pMtENOD20-GUS fusion is first activated during the earliest stages of nodule organogenesis in the alfalfa root inner cortex, approximately $24 \mathrm{~h}$ following $S$. meliloti inoculation, and we have used RT-PCR to confirm that the endogenous MtENOD20 gene follows similar activation kinetics. Analysis of histochemically stained tissues has revealed a strict correlation between reporter gene activity and the presence of cell divisions in the inner cortex, corresponding to sites of nodule primordium formation (Fig. 1B), although at this stage we have no information concerning the precise temporal relationship between cell division and gene expression.

In addition to nodule primordium-related gene expression, reporter gene activity was also detected in the root epidermis at sites of primordium development (Fig. 1C and D). GUS staining appeared to be strictly limited to root hairs containing infection threads and our results with $S$. meliloti nodulation mutants and purified Nod factors together suggest that MtENOD20 expression in root hairs is indeed infection dependent (Fig. 3A-C). The relative timing of MtENOD2O activation in the cortex and epidermis is in line with previous observations that CCA precedes root hair infection (Calvert et al. 1984; Dudley et al. 1987; Bauchrowitz et al. 1996).

It should be recalled that the MtENOD20 gene encodes a protein that belongs to a small family of early nodulins (including MtENOD16, Ps/VsENOD5, and GmN315/ ENOD55) with structural (but probably not functional) homology to phytocyanins (Greene et al. 1998). In situ hybridization studies have shown that the Ps/VsENOD5 genes are expressed in root cortical cells carrying infection threads and in infected cells of the nodule, but not in the nodule pri-
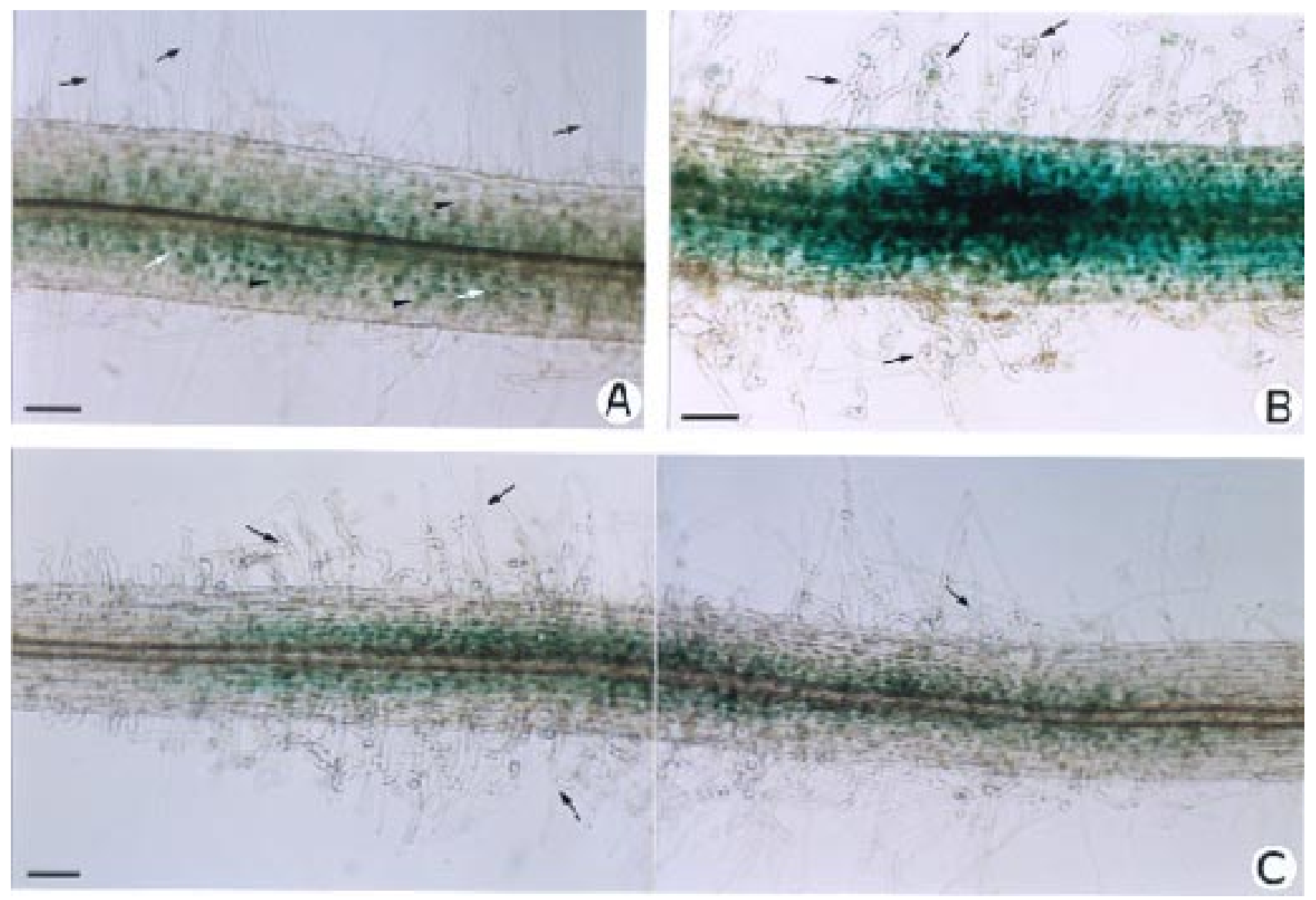

Fig. 3. Localization of $\beta$-glucuronidase (GUS) activity in transgenic Medicago varia roots in response to either Sinorhizobium meliloti nod mutants or purified Nod factors. A, Root segment of transgenic M. varia $72 \mathrm{~h}$ post inoculation with RCR2011 nodH::Tn5. Anticlinal cell divisions in the inner cortex (white arrows), and centrally located nuclei with surrounding amyloplasts (arrowheads) are typical characteristics of cortical cell activation (CCA; Ardourel et al. 1994). Dark arrows indicate undamaged root hairs above the zone of CCA that are not deformed. Bar $=100 \mu \mathrm{m}$. B, Transgenic $M$. varia root 4 days post inoculation with RCR2011 $\Delta$ nodF/nodL::Tn5. Cortical GUS staining associated with CCA is very intense, and root hairs exhibit the striking deformations typical for this mutant (arrows). Bar $=100 \mu \mathrm{m}$. C, Root segment showing a complete zone of GUS staining $48 \mathrm{~h}$ after the addition of $10^{-8} \mathrm{M}$ NodRm to transgenic $M$. varia. Reporter gene activity is localized essentially to the inner cortex. Unstained root hairs throughout the entire zone (but not outside) display characteristic Nod-factor elicited deformations such as branching (arrows). Bar $=100 \mu \mathrm{m}$. 
mordium prior to infection (Scheres et al. 1990; Vijn et al. 1995). We have reached similar conclusions for the MtENOD16 gene based on a transgenic plant/reporter gene approach (V. Vernoud, unpublished results). The soybean N315/ENOD55 genes are also expressed in infected cells of the nodule (de Blank et al. 1993; Kouchi and Hata 1993). These findings suggest that the expression of all members of this gene family can be correlated with developmental events related to host cell infection by the microsymbiont. On the other hand, MtENOD20 is the only gene member identified so far that is also activated during nodule organogenesis in the root cortex.

We have previously proposed that the extracellular proteins encoded by this gene family may be components of the cell wall matrix, with possible roles in cell wall assembly/reorganization (Greene et al. 1998). Such a function would certainly be compatible with both the requirements for infection thread progression/penetration and the likely restructuring of the cell wall in the developing primordium. All members of this early nodulin family possess a Pro-rich domain, highly variable in length, sequence, and composition, located on the C-terminal side of the conserved globular domain. However, it is striking that MtENOD20 possesses by far the largest (hydroxy)proline-rich domain of the entire family, consisting essentially of hydroxyproline-rich plant glycoprotein (HRGP) structural motifs (Greene et al. 1998). It remains to be determined whether there is a relationship between this lengthy HRGPlike domain and the unique expression pattern of the MtENOD20 gene.

\section{MtENOD20 gene expression is associated with CCA in the absence of infection.}

Symbiotic MtENOD20 expression has been further investigated in experiments exploiting $S$. meliloti nod mutants with nodulation-defective phenotypes resulting from structural modifications to the Nod factor. The $S$. meliloti nodF/nodL mutant produces Nod factors (NodRm-IV[S,C18:1]) modified at the nonreducing end of the sugar backbone and is infectiondefective (Ardourel et al. 1994). However, this mutant is still able to elicit both elaborate root hair deformations and a striking cortical cell response characterized by starch accumulation, the distribution of amyloplasts around cortical cell nuclei, and limited cell divisions, that has been termed cortical cell activation (CCA). The pMtENOD20-GUS fusion is strongly expressed at sites of CCA resulting from inoculation of transgenic alfalfa with a nodF/nodL mutant (Fig. 3B). This is consistent with activation of the transgene during early stages of nodule primordium development (see above) and also at sites of CCA elicited by both the S. meliloti nodH mu-

Table 2. Percentage of $\beta$-glucuronidase (GUS)-positive plants as a function of the concentration of exogenously Sinorhizobium meliloti Nod factor

\begin{tabular}{lcccc}
\hline \multirow{2}{*}{$\begin{array}{l}\text { NodRm } \\
\text { concentration }^{\mathbf{n}}(\mathbf{M})\end{array}$} & \multicolumn{4}{c}{ Length of Nod factor treatment (days) } \\
\cline { 2 - 5 } & $\mathbf{1}$ & $\mathbf{2}$ & $\mathbf{3}$ & $\mathbf{5}$ \\
\hline $10^{-7}$ & 80 & 90 & 100 & 100 \\
$10^{-8}$ & 60 & 80 & 100 & 100 \\
$10^{-9}$ & 60 & 80 & 90 & 90 \\
$10^{-10}$ & 40 & 70 & 80 & 80 \\
$10^{-11}$ & 30 & 50 & 60 & 60 \\
$10^{-12}$ & 0 & 0 & 0 & 0 \\
\hline
\end{tabular}

tant and purified Nod factors (Fig. 3A and C, respectively; see below). In this paper, RT-PCR experiments have been used to demonstrate rapid induction of the endogenous MtENOD2O gene in response to both $S$. meliloti inoculation and purified Nod factor treatment (Fig. 2). Although RT-PCR was not used to confirm endogenous MtENOD2O expression elicited by the nodF/nodL and nodH mutants, it is clear from the use of histochemical GUS localization that pMtENOD20-GUS expression in the inner cortex is always associated with differentiation processes related to symbiotic CCA.

$S$. meliloti nodH mutants synthesize Nod factors that lack the terminal reducing end $O$-sulfate substitution (Roche et al. 1991) and until now have been considered unable to elicit symbiotic responses on the normal host plant alfalfa. However, we have discovered that nodH mutants are capable of activating MtENOD2O expression coupled to CCA (Fig. 3A), although less efficiently than the nodF/nodL mutant. It is probable that this symbiotic response was not previously reported for nodH mutants (Truchet et al. 1991; Ardourel et al. 1994) because of both its relatively low frequency and the limited extent of CCA, which never develops as far as visible root swellings. On the other hand, purified non-sulfated $S$. meliloti factors were not able to elicit MtENOD20 expression and the CCA response in transgenic alfalfa lines, even when applied at relatively high concentrations (up to $10^{-7} \mathrm{M}$ ). One possible explanation for this apparent paradox is that the nodH mutant synthesizes a second molecule that is either sufficient itself for low-level CCA stimulation, or acts in synergy with the nonsulfated Nod factor. A somewhat analogous situation has been described for Bradyrhizobium japonicum nodZ mutants that produce nonfucosylated, pentameric Nod factors that lack biological activity, and yet nodulate soybean normally (Stacey et al. 1994; Stokkermans et al. 1995). However, it is also possible that plant chitinases are responsible for the failure of exogenously supplied, nonsulfated factors to elicit CCA, since Staehelin et al. (1994) have demonstrated that the absence of the sulfate group makes the $S$. meliloti Nod factors particularly sensitive to chitinase degradation. Furthermore, Goormachtig et al. (1998) have recently identified a Sesbania rostrata chitinase-encoding gene that is transcribed very early during nodulation in cortical tissues adjacent to the developing primordium. If the chitinase hypothesis is correct, then the ability of the $n o d H$ mutant to initiate CCA suggests that the continued synthesis of Nod factors by the bacterium at the root surface is necessary for this activity.

Significantly, root hair deformations are not observed in epidermal tissues above sites of nodH mutant-elicited CCA

Table 3. Percentage of $\beta$-glucuronidase (GUS)-positive plants as a function of lipo-chitooligosaccharide (LCO) structure

\begin{tabular}{lccc}
\hline & \multicolumn{3}{c}{$\begin{array}{c}\text { Length of elicitor } \\
\text { treatment (days) }\end{array}$} \\
\cline { 2 - 4 }${\text { Elicitor }\left(\mathbf{1 0}^{-\mathbf{8}} \mathbf{M}\right)^{\mathbf{a}}}^{\mathbf{2}}$ & $\mathbf{2}$ & $\mathbf{3}$ & $\mathbf{5}$ \\
\hline NodRm-IV(Ac, S, C16:2) (parental strain) & 80 & 100 & 100 \\
NodRm-IV(Ac, C16:2) (nodH mutant) & 0 & 0 & 0 \\
NodRm-IV(S, C18:1) (nodF/nodL mutant) & 80 & 90 & 90 \\
LCO-IV(S, C16:1) & 70 & 90 & 90 \\
LCO-IV(S, C12:1) & 70 & 80 & 80 \\
LCO-IV(S, C8:1) & 30 & 30 & 50 \\
CO-IV(S, $\left.\mathrm{NH}_{2}\right)$ & 0 & 0 & 0 \\
\hline
\end{tabular}

${ }^{a}$ Abbreviations: Ac, $O$-acetyl; $\mathrm{S}, O$-sulfate. 
(Fig. 3A), in line with previous reports that the nodH mutant is unable to induce symbiotic epidermal responses (Debellé et al. 1986), including expression of the MtENOD12 gene (Journet et al. 1994). Assuming that the CCA activity of the nodH mutant is due to the nonsulfated factor, this suggests that the absence of the sulfate moiety is more critical for the epidermal response. These results also imply that root cortical responses can be elicited without apparent activation of epidermal tissues and therefore favor the hypothesis that different Nod factor perception mechanisms are involved in initiating early symbiotic responses in the cortex and epidermis.

\section{MtENOD20 as a molecular marker \\ for Nod factor-elicited CCA.}

When purified NodRm factor is added to transgenic alfalfa roots $\mathrm{p} M t E N O D 20-G U S$ expression associated with localized sites of CCA can be detected within 24 h (Fig. 3C). As with $S$. meliloti-elicited expression, we have confirmed the rapid induction of endogenous MtENOD20 transcripts with RT-PCR amplification of total RNA extracted from Nod factor-treated $M$. truncatula roots (Fig. 2B). Use of transgenic plants has further demonstrated that MtENOD2O expression linked to CCA can be detected at NodRm concentrations as low as $10^{-11}$ M (Table 2). We have previously proposed that the physiological concentration of Nod factors produced by the microsymbiont at the root surface is most probably in the subnanomolar range (Journet et al. 1994). If this is indeed the case then our results show for the first time that exogenously applied Nod factors are capable of initiating CCA at physiological levels.

We have made use of a series of synthetic, non-Oacetylated LCOs with variable acyl chain lengths to show that the length of the lipid chain is of critical importance for eliciting gene activation coupled to CCA in the cortex. Even at the relatively high concentration of $10^{-8} \mathrm{M}$, shortening the fatty acid to half the normal length (C8:1) results in a major reduction in CCA activity, while nonacylated, sulfated chitin tetramers are totally devoid of activity (Table 3 ). These findings are consistent with a recent Nod factor structure/activity study employing the same synthetic LCOs and alfalfa nodulation as a semiquantitative assay (Demont-Caulet et al., in press). Our experiments with biological (NodRmIV[S,C18:1]; nodF/ nodL mutant) and synthetic (LCOIV [S,C16:1]) molecules show that, when tested at $10^{-8} \mathrm{M}$, removal of the $O$-acetyl group and modifications to the number and position of the double bonds within the $N$-acyl chain have no detectable effect on the efficiency of CCA induction (Table $3)$. Nevertheless, it will be important to repeat these experiments when synthetic, O-acetylated LCOs become available and to use a lower range of LCO concentrations in order to increase the sensitivity of the assay.

These findings for alfalfa are in line with previous reports showing that the exogenous addition of either unsubstituted or O-acetylated chitin oligomers to vetch and soybean fails to elicit cortical cell divisions and nodule primordium formation (Spaink et al. 1991; Minami et al. 1996; Schlaman et al. 1997). However, when microtargeted into the cortex, Oacetylated chitin oligomers appear to be capable of eliciting changes in the root auxin balance characteristic of nodule initiation in clover (Mathesius et al. 1998) and inducing cortical cell divisions in vetch in the presence of uridine (Schlaman et al. 1997). Taken together, these findings imply that the fatty acyl moiety may be necessary for LCO transport across the epidermal cell layer, and are consistent with different sites of perception for Nod factor-elicited epidermal and cortical responses, as discussed above.

MtENOD20 now joins a small group of early nodulin genes including ENOD4O (Vijn et al. 1995; Minami et al. 1996; Fang and Hirsch 1998), Mtlecl\&3 (Bauchrowitz et al. 1996), and certain ENOD12 genes (Vijn et al. 1995; Bauer et al. 1996) whose nodulation-related expression in cortical tissues can be elicited by both Rhizobium and purified Nod factors. However, compared with that of the majority of the other early nodulins, MtENOD20 gene expression within the root can be considered essentially specific to the nodulation process. This is illustrated both by the absence of detectable MtENOD20 transcription in noninoculated $M$. truncatula roots (even after 38 cycles of RT-PCR) and the specificity of GUS staining with respect to symbiotically activated tissues in transgenic $M$. varia roots (with the exception of a low background vascular staining for a minority of pouch-grown plants). Analogous transgenic plant/reporter gene studies have shown that MsENOD40 and MsENOD12A genes are expressed in lateral root primordia (Bauer et al. 1996; Fang and Hirsch 1998) and Mtlecl\&3 in inner root tissues independently of nodulation (Bauchrowitz et al. 1996).

Until now the organogenetic activity of Nod factors in the majority of legumes has generally been studied by means of cytological analyses. This approach often requires lengthy (1 to 3 weeks) exposures to high concentrations of Nod factor, and is particularly difficult to exploit when the resulting CCA is limited to only a few rounds of division. The results presented in this article indicate that transgenic plants expressing the pMtENOD20-GUS fusion should provide both a sensitive and rapid assay for monitoring Nod factor-elicited CCA, and at the same time a convenient marker for distinguishing between root and nodule primordia. The identical transgene has recently been introduced into the homologous host $M$. truncatula in order to facilitate the detailed characterization of plant mutants that are defective in early stages of nodulation.

\section{MATERIALS AND METHODS}

\section{Construction of a transcriptional MtENOD20 promoter- GUS fusion.}

In order to create a precise transcriptional fusion between the promoter region of the MtENOD20 gene and the coding region of the Escherichia coli GUS reporter gene, a PCR strategy similar to that described in Pichon et al. (1992) was used. Briefly, a genomic MtENOD20 clone (Greene et al. 1998) was used as template for PCR with a forward primer (5'-CTACTA $\underline{A} G C T T(E c o$ RI)CTTCGGTGAAGACC-3') and reverse primer (5'-AGAAG $\underline{C C} \underline{\underline{A T G}} \underline{G}(\mathrm{NcoI}) \mathrm{TCATGATTCT}-$ $3^{\prime}$ ) in order to amplify a 2.15 -kb promoter DNA fragment (single underlined bases correspond to substitutions required to create the labeled restriction sites and double underlining indicates the MtENOD2O translation initiation codon). After digestion with EcoRI and NcoI the MtENOD2O promoter fragment was cloned between the EcoRI and NcoI sites within the polylinker region of the vector pBSGUS, in order to generate a precise transcriptional fusion. The vector pBSGUS is a pBluescript hybrid that was constructed by cloning the 
EcoRI/PstI GUS coding fragment from pCCOGUS (Axelos et al., 1989) into the corresponding polylinker sites of pBluescript $\mathrm{KS}^{+}$(Stratagene, La Jolla, CA). The

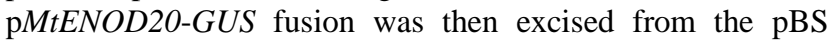
vector by HindIII/SacI digestion, and cloned between the corresponding unique sites in the binary vector pLP100 (Szabados et al. 1995). The junction between the promoter fragment and the GUS coding region was confirmed by DNA sequencing.

\section{Transformation of $M$. varia and regeneration of transgenic plants.}

The introduction of the pMtENOD20-GUS fusion into the $M$. varia genotype A2 (Deak et al. 1986) was performed essentially as described in Pichon et al. (1992). Scarified leaflets were co-cultivated for 4 days with Agrobacterium tumefaciens LBA4404 containing the pLP100-pMtENOD20-GUS binary vector. Somatic embryogenesis was induced on kanamycinresistant callus, with the first embryos appearing 1 month after co-culture. For maturation and plantlet formation, embryos were transferred to hormone-free medium supplemented with maltose, proline, and charcoal. Regenerated primary transformants, each derived from an individual leaf callus, were grown on axenic medium containing $1 \%$ sucrose at $25^{\circ} \mathrm{C}$ with a 16-h photoperiod $\left(65 \mu \mathrm{E} \mathrm{s}^{-1} \mathrm{~m}^{-2}\right)$ and were propagated by cuttings. To obtain transgenic $S_{1}$ seeds, primary transformants were transferred to pots and grown under the appropriate conditions to induce flowering and pod formation $\left(25^{\circ} \mathrm{C}, 16-\mathrm{h}\right.$ photoperiod, $230 \mu \mathrm{E} \mathrm{s}^{-1} \mathrm{~m}^{-2}$ ).

\section{S. meliloti inoculation and Nod factor addition to transgenic plants.}

For reporter gene expression studies during symbiosis, cuttings of primary $M$. varia transformants were grown under aeroponic conditions as described in Bauchrowitz et al. (1996). After 2 to 3 weeks of growth, combined nitrogen was removed from the medium and plants were inoculated with the $S$. meliloti strain RCR2011 (approximately $5 \times 10^{5}$ bacteria per $\mathrm{ml}$ ). Whole root segments or nodules were collected at various times after inoculation for GUS histochemical staining (see below).

Transgenic $S_{1}$ seeds from two representative primary transformant lines were used both for experiments with $S$. meliloti symbiotic mutants and for tests with purified Nod factors or synthetic LCOs. Seeds were surface sterilized (8 min $\mathrm{H}_{2} \mathrm{SO}_{4}$, followed by 2 min $12 \%$ sodium hypochlorite) and germinated for $36 \mathrm{~h}$ in the dark at $25^{\circ} \mathrm{C}$. Transgenic seedlings (five plants per pouch) were then placed in plastic growth pouches (Mega International, Minneapolis) as described in Journet et al. (1994). Following the development of secondary roots (7 to 10 days later), roots were either inoculated with $S$. meliloti (approximately $5 \times 10^{6}$ bacteria per root) or treated with the appropriate Nod factor/LCO by direct addition into the growth pouch.

\section{Bacterial strains, purified Nod factors, and synthetic LCOs.}

Transgenic plants in pouches were inoculated with the parental S. meliloti strain RCR2011 and with the following four mutant strains: RCR2011 nodA::Tn5\#2208 (GMI 5383, Debellé et al. 1986), RCR2011 nodH::Tn5\#2313 (GMI5431, De- bellé et al. 1986), RCR2011 $\Delta($ nodH)8 (GMI5619, Debellé et al. 1988), RCR2011 $\Delta$ (nodF)13nodL::Tn5 (GMI6628, Ardourel et al. 1994). S. meliloti strains were grown at $28^{\circ} \mathrm{C}$ in standard TY medium supplemented with $6 \mathrm{mM} \mathrm{CaCl}_{2}$ and appropriate antibiotic selection.

$S$. meliloti Nod factors purified from the corresponding overproducing parental and mutant strains (Roche et al. 1991) were kindly provided by F. Maillet (J. Dénarié's group, LBMRPM, Castanet-Tolosan). Sulfated LCO-IV with variable length fatty acid chains (C8:1, C12:1, and C16:1) and sulfated chitin tetramer lacking the acyl chain and with a free $\mathrm{NH}_{2}$ $\left[\mathrm{CO}-\mathrm{IV}\left(\mathrm{S}, \mathrm{NH}_{2}\right)\right]$ were a kind gift of J.-M. Beau and were synthesized as described in Demont-Caulet et al. (in press). Serial dilutions of Nod factors and LCOs were carried out in sterile water.

\section{Histochemical localization of GUS activity and the semiquantitative assay.}

Histochemical staining for GUS activity was performed on whole root segments for up to $24 \mathrm{~h}$ with the substrate X-Gluc (5-bromo-4-chloro-3-indolyl glucuronide, cyclohexylammonium salt; Biosynth AG, Staad, Switzerland) as described in Journet et al. (1994). Nodules were sliced into 80- $\mu$ m-thick sections (Microcut H1200; Bio-Rad, Hercules, CA) prior to staining for GUS activity. In this case, prefixation of intact samples was carried out with $0.5 \%$ para-formaldehyde and incubation times in GUS reaction medium were generally limited to between $15 \mathrm{~min}$ and $2 \mathrm{~h}$. Stained intact samples or slices were briefly cleared with sodium hypochlorite as described in Pichon et al. (1992) and observed with a Zeiss Axiophot light microscope (Carl Zeiss, Oberkochen, Germany). For localization of GUS activity at the cellular level, stained roots were post-fixed for $1 \mathrm{~h}$ in $2.5 \%$ glutaraldehyde buffered in $0.1 \mathrm{M}$ potassium phosphate $(\mathrm{pH} 7.0)$, dehydrated in an alcohol series and embedded in Technovit 7100 resin (Heraeus Kulzer, Wehrheim, Germany). Sections ( $4 \mu \mathrm{m}$ thick) were observed by bright- and dark-field microscopy after counterstaining with basic fuchsin $(0.5 \%$ in distilled water). Double staining for GUS and $\beta$-galactosidase activity after inoculation with a RCR2011 strain carrying a constitutive hemA-lacZ fusion (Ardourel et al. 1994) was performed according to Pichon et al. (1994).

In our semiquantitative assay, transgenic plants were scored positive for GUS staining in the cortex on the basis of a clear and unambiguous signal located at or below the region of the root that is normally susceptible to nodulation at the moment of bacterial inoculation. This susceptible region lies between the root tip and the zone of root hair emergence (Bhuvaneswari et al. 1981; Caetano-Anollés and Gresshoff 1991), and the use of growth pouches enabled us to monitor root growth following either $S$. meliloti inoculation or Nod factor addition and thus to position GUS expression in relation to the susceptible zone. This approach eliminated the potential risk of confusing Rhizobium/Nod factor responses with the development of spontaneous nodule primordia in the older region of the root (see Pichon et al. 1994). Since approximately $20 \%$ of the transgenic alfalfa $S_{1}$ plants generated after selfing were GUS negative, the percentage values presented in Tables 1-3 have been corrected to take this into account. At least 15 to 20 plants were tested for each experimental condition, and each series of experiments was repeated at least 
twice. In the experiments with nodH mutants, a total of over 150 plants were tested. Percent values have been rounded up to the nearest $10 \%$ and statistical analysis has shown that, in the majority of cases, differences of $10 \%$ cannot be considered significant.

\section{RT-PCR assay to detect $M t E N O D 20$ transcripts.}

For each time point $(0,12,24,48$, and $72 \mathrm{~h})$, secondary roots of 30 pouch-grown $M$. truncatula cv. Jemalong plants, either inoculated with $S$. meliloti 2011 or treated with purified Nod factors, were harvested and ground in liquid nitrogen. Total RNA was isolated by a standard phenol/sodium dodecyl sulfate protocol and quantified by spectrophotometry and agarose gel electrophoresis. Prior to RT-PCR, genomic DNA was eliminated by treating 1 - to 5- $\mu$ g aliquots of total RNA with 10 $\mathrm{U}$ of DNase I in RT buffer $(50 \mathrm{mM}$ TrisHCl, $75 \mathrm{mM} \mathrm{KCl,} 3$ $\mathrm{mM} \mathrm{MgCl}_{2}, \mathrm{pH} 8.3$ ) at $37^{\circ} \mathrm{C}$ during $30 \mathrm{~min}$, followed by phenol/chloroform extraction and ethanol precipitation. Firststrand cDNA synthesis with 1 to $3 \mu \mathrm{g}$ of total RNA was performed as described in Pingret et al. (1998). Primers were chosen on either side of the single MtENOD20 intron (Greene et al. 1998) in order to distinguish cDNA from genomic amplified products. A 283-bp MtENOD20 cDNA fragment was amplified during 38 cycles $\left(94^{\circ} \mathrm{C} / 30 \mathrm{~s}, 62^{\circ} \mathrm{C} / 30 \mathrm{~s}, 72^{\circ} \mathrm{C} / 30 \mathrm{~s}\right)$ between positions 123 and 570 (relative to the ATG codon) of the genomic sequence (GenBank accession no. X99467) with forward primer 5'-AACTCGACATGCACTCACCCG-3', and reverse primer 5'-GGAAGAAAGTACGGGAGCTACC-3'. Under our experimental conditions an exponential increase of PCR product was still observed after 38 cycles. The parallel amplification of a constitutively expressing gene in roots, MtPR10-1 (Gamas et al. 1998; GenBank accession no. Y08726), allowed us to control for equivalent cDNA levels in samples. MtPR10-1 cDNA was amplified during 25 cycles according to Pingret et al. (1998). PCR products were analyzed by Southern blotting with the $1-\mathrm{kb}$ HindIII/SstI fragment of MtENOD2O and the complete cDNA for MtPR10-1.

\section{ACKNOWLEDGMENTS}

We would like to thank Annie Dedieu for technical assistance during the construction of the pMtENOD20-GUS fusion, Jean-Luc Pingret for his guidance in setting up the RT-PCR assay, and David Villa for his help with the illustrations. We are also grateful to Fabienne Maillet and Jean Dénarié for providing purified biological Nod factors and to JeanMarie Beau for the synthetic LCOs. Finally, thanks to the many colleagues who contributed constructive criticisms during the preparation of the manuscript, and in particular Fernanda de Carvalho-Niebel, JeanLuc Pingret, and Georges Truchet. Financial support at the outset of the project was provided by the European Community Biotechnology Program, as part of the Project of Technological Priority 1993-1996. V. V. received a grant from the French Ministère de l'Enseignement Supérieur et de la Recherche.

\section{LITERATURE CITED}

Ardourel, M., Demont, N., Debellé, F., Maillet, F., de Billy, F., Promé, J.-C., Dénarié, J., and Truchet, G. 1994. Rhizobium meliloti lipooligosaccharide nodulation factors: Different structural requirements for bacterial entry into target cells and induction of plant symbiotic developmental processes. Plant Cell 6:1357-1374.

Axelos, M., Bardet, C., Le Van Thai, A., Curie, C., and Lescure, B. 1989. The family encoding the Arabidopsis thaliana elongation factor
EF-1 $\alpha$ : Molecular cloning, characterization and expression. Mol. Gen. Genet. 219:106-112.

Bauer, P., Ratet, P., Crespi, M. D., Schultze, M., and Kondorosi, A. 1996. Nod factors and cytokinins induce similar cortical cell division, amyloplast deposition and MsEnod $12 \mathrm{~A}$ expression patterns in alfalfa roots. Plant J. 10:91-105.

Bauchrowitz, M. A., Barker, D. G., and Truchet, G. 1996. Lectin genes are expressed throughout root nodule development and during nitrogen-fixation in the Rhizobium-Medicago symbiosis. Plant J. 9:31-43.

Bhuvaneswari, T. V., Bhagwat, A. A. and Bauer, W. D. 1981. Transient susceptibility of root cells in four common legumes to nodulation by rhizobia. Plant Physiol. 68:1144-1149.

Caetano-Anollés, G., and Gresshoff, P. M. 1991. Alfalfa controls nodulation during the onset of Rhizobium-induced cortical cell division. Plant Physiol. 95:366-373.

Calvert, H. E., Pence, M. K., Pierce, M., Malik, N. S. A., and Bauer, W. D. 1984. Anatomical analysis of the development and distribution of Rhizobium infections in soybean roots. Can. J. Bot. 30:2375-2384.

de Blank, C., Mylona, P., Yang, W. C., Katinakis, P., Bisseling, T., and Franssen, H. 1993. Characterization of the soybean early nodulin cDNA clone GmENOD55. Plant Mol. Biol. 22:1167-1171.

Deak, M., Kiss, G. B., Koncz, C., and Dudits, D. 1986. Transformation of Medicago by Agrobacterium mediated gene transfer. Plant Cell Rep. 5:97-100.

Debellé, F., Maillet, F., Vasse, J., Rosenberg, C., de Billy, F., Truchet, G., Dénarié, J., and Ausubel, F. M. 1988. Interference between Rhizobium meliloti and Rhizobium trifolii nodulation genes: Genetic basis of $R$. meliloti dominance. J. Bacteriol. 170:5718-5727.

Debellé, F., Rosenberg, C., Vasse, J., Maillet, F., Martinez, E., Dénarié, J., and Truchet, G. 1986. Assignment of symbiotic developmental phenotypes to common and specific nodulation (nod) genetic loci of Rhizobium meliloti. J. Bacteriol. 168:1075-1086.

Demont-Caulet, N., Maillet, F., Tailler, D., Jacquinet, J.-C., Promé, J.-C., Nicolaou, K. C., Truchet, G., Beau, J.-M., and Dénarié, J. Noduleinducing activity of synthetic Sinorhizobium meliloti Nod factors and related lipo-oligosaccharides on Medicago sativa: Importance of the acyl chain structure. Plant Physiol. (In press.)

Dénarié, J., Debellé, F., and Promé, J.-C. 1996. Rhizobium lipochitooligosaccharide nodulation factors: Signaling molecules mediating recognition and morphogenesis. Annu. Rev. Biochem. 65:503535.

Dudley, M. E., Jacobs, T. W., and Long, S. R. 1987. Microscopic studies of cell divisions induced in alfalfa roots by Rhizobium meliloti. Planta 171:289-301.

Fang, Y., and Hirsch, A. M. 1998. Studying early nodulin gene ENOD40 expression and induction by nodulation factor and cytokinin in transgenic alfalfa. Plant Physiol. 116:53-68.

Gamas, P., de Billy, F., and Truchet, G. 1998. Symbiosis-specific expression of two Medicago truncatula nodulin genes, MtN1 and MtN13, encoding products homologous to plant defense proteins. Mol. PlantMicrobe Interact. 11:393-403.

Goormachtig, S., Lievens, S., van de Velde, W., van Montagu, M., and Holsters, M. 1998. Srchi13, a novel early nodulin gene from Sesbania rostrata, is related to acidic class III chitinases. Plant Cell 10:905915.

Greene, E. A., Erard, M., Dedieu, A. and Barker, D. G. 1998. MtENOD16 and 20 are members of a family of phytocyanin-related early nodulins. Plant Mol. Biol. 36:775-783.

Heidstra, R., and Bisseling, T. 1996. Nod factor-induced host responses and mechanisms of Nod factor perception. New Phytol. 133:25-43.

Journet, E.-P., Pichon, M., Dedieu, A., de Billy, F., Truchet, G., and Barker, D. G. 1994. Rhizobium meliloti Nod factors elicit cell-specific transcription of the ENOD12 gene in transgenic alfalfa. Plant J. 6:241249.

Kouchi, H., and Hata, S. 1993. Isolation and characterization of novel nodulin cDNAs representing genes expressed at early stages of soybean nodule development. Mol. Gen. Genet. 238:106-119.

Lerouge, P., Roche, P., Faucher, C., Maillet, F., Truchet, G., Promé, J.C., and Dénarié, J. 1990. Symbiotic host-specificity of Rhizobium meliloti is determined by a sulfated and acylated glucosamine oligosaccharide signal. Nature 344:781-784.

Long, S. R. 1996. Rhizobium symbiosis: Nod factors in perspective. Plant Cell 8:1885-1898.

Mathesius, U., Schlaman, H. R. M., Spaink, H. P., Sautter, C., Rolfe, B. 
G., and Djordjevic, M. A. 1998. Auxin transport inhibition precedes root nodule formation in white clover roots and is regulated by flavonoids and derivatives of chitin oligosaccharides. Plant J. 14:23-34.

Minami, E., Kouchi, H., Cohn, J. R., Ogawa, T., and Stacey, G. 1996. Expression of the early nodulin, ENOD40, in soybean roots in response to various lipo-chitin signal molecules. Plant J. 10:23-32.

Penmetsa, R. V., and Cook, D. R. 1997. A legume ethylene-insensitive mutant hyperinfected by its rhizobial symbiont. Science 275:527-530.

Pichon, M., Journet, E.-P., de Billy, F., Dedieu, A., Huguet, T., Truchet, G., and Barker, D. G. 1994. ENOD12 gene expression as a molecular marker for comparing Rhizobium-dependent and -independent nodulation in alfalfa. Mol. Plant-Microbe Interact. 7:740-747.

Pichon, M., Journet, E.-P., Dedieu, A., de Billy, F., Truchet, G., and Barker, D. G. 1992. Rhizobium meliloti elicits transient expression of the early nodulin gene ENOD12 in the differentiating root epidermis of transgenic alfalfa. Plant Cell 4:1199-1211.

Pingret, J.-L., Journet, E.-P., and Barker, D. G. 1998. Rhizobium Nod factor signaling: Evidence for a $\mathrm{G}$ protein-mediated transduction mechanism. Plant Cell 10:659-671.

Roche, P., Debellé, F., Maillet, F., Lerouge, P., Faucher, C., Truchet, G., Dénarié, J., and Promé, J.-C. 1991. Molecular basis of symbiotic host specificity in Rhizobium meliloti: nodH and nodPQ genes encode the sulfation of lipo-oligosaccharide signals. Cell 67:1131-1143.

Rosenberg, C., Boistard, P., Dénarié, J., and Casse-Delbart, F. 1981. Genes controlling early and late functions in symbiosis are located on a megaplasmid in Rhizobium meliloti. Mol. Gen. Genet. 184:326-333.

Scheres, B., van Engelen, F., van der Knaap, E., van de Wiel, C., van Kammen, A., and Bisseling T. 1990. Sequential induction of nodulin gene expression in the developing pea nodule. Plant Cell 2:687-700.

Schlaman, H. R. M., Gisel, A. A., Quaedvlieg, N. E. M., Bloemberg, G. V., Lugtenberg, B. J. J., Kijne, J. W., Potrykus, I., Spaink, H. P., and Sautter, C. 1997. Chitin oligosaccharides can induce cortical cell division in roots of Vicia sativa when delivered by ballistic microtargeting. Development 124:4887-4895.
Spaink, H. P., Sheeley, D. M., van Brussel, A. A. N., Glushka, J., York, W. S., Tak, T., Geiger, O., Kennedy, E. P., Reinhold, V. N., and Lugtenberg, B. J. J. 1991. A novel highly unsaturated fatty acid moiety of lipo-oligosaccharide signals determines host specificity of Rhizobium. Nature 354:125-130.

Stacey, G., Luka, S., Sanjuan, J., Banfalvi, Z., Niewkoop, A. J., Chun, J. Y., Forsberg, L. S., and Carlson, R. 1994. nodZ, a unique host-specific nodulation gene, is involved in the fucosylation of the lipooligosaccharide nodulation signal of Bradyrhizobium japonicum. J. Bacteriol. 176:620-633.

Staehelin, C., Schultze, M., Kondorosi, E., Mellor, R. B., Boller, T., and Kondorosi, A. 1994. Structural modifications in Rhizobium meliloti Nod factors influence their stability against hydrolysis by root chitinases. Plant J. 5:319-330.

Stokkermans, T. J. W., Ikeshita, S., Cohn, J., Carlson, R. W., Stacey, G., Ogawa, T., and Peters, N. K. 1995. Structural requirements of synthetic and natural product lipo-chitin oligosaccharides for induction of nodule primordia on Glycine soja. Plant Physiol. 108:1587-1595.

Szabados, L., Charrier, B., Kondorosi, A., de Bruijn, F. J., and Ratet, P. 1995. New plant promoter and enhancer testing vectors. Mol. Breeding 1:419-425.

Truchet, G., Roche, P., Lerouge, P., Vasse, J., Camut, S., de Billy, F., Promé, J.-C., and Dénarié, J. 1991. Sulfated lipo-oligosaccharide signals of Rhizobium meliloti elicit root nodule organogenesis in alfalfa. Nature 351:670-673.

Vasse, J., de Billy, F., Camut, S., and Truchet, G. 1990. Correlation between ultrastructural differentiation of bacteroids and nitrogen fixation in alfalfa nodules. J. Bacteriol. 172:4295-4306.

Vasse, J., de Billy, F., and Truchet, G. 1993. Abortion of infection during the Rhizobium meliloti-alfalfa symbiotic interaction is accompanied by a hypersensitive reaction. Plant J. 4:555-566.

Vijn, I., Martinez-Abarca, F., Yang, W.-C., das Neves, L., van Brussel, A., van Kammen, A., and Bisseling, T. 1995. Early nodulin gene expression during Nod factor-induced processes in Vicia sativa. Plant J. 8:111-119. 